\title{
Systematic Problem Solving in Production: The NAX Approach
}

\author{
Axelsdottir, Aslaug; Nygaard, Martin; Edwards, Kasper
}

Published in:

I E E E Engineering Management Review

Link to article, DOI:

10.1109/EMR.2017.2667178

Publication date:

2017

Document Version

Peer reviewed version

Link back to DTU Orbit

Citation (APA):

Axelsdottir, A., Nygaard, M., \& Edwards, K. (2017). Systematic Problem Solving in Production: The NAX Approach. I E E E Engineering Management Review, 45(1), 49-57. https://doi.org/10.1109/EMR.2017.2667178

\section{General rights}

Copyright and moral rights for the publications made accessible in the public portal are retained by the authors and/or other copyright owners and it is a condition of accessing publications that users recognise and abide by the legal requirements associated with these rights.

- Users may download and print one copy of any publication from the public portal for the purpose of private study or research.

- You may not further distribute the material or use it for any profit-making activity or commercial gain

- You may freely distribute the URL identifying the publication in the public portal

If you believe that this document breaches copyright please contact us providing details, and we will remove access to the work immediately and investigate your claim 


\section{Systematic Problem Solving in Production: The NAX Approach}

Aslaug Axelsdottir, Martin Nygaard and Kasper Edwards (corresponding author, kaed@dtu.dk)

Abstract-This paper outlines the NAX problem solving approach developed by a group of problem solving experts at a large Danish Producer of medical equipment. The company, "Medicmeter" is one of Denmark’s leading companies when it comes to lean and it has developed a strong problem solving culture. The main steps of the approach are to extensively gather direct detailed process knowledge at the actual process, assemble a team that systematically builds on each other ideas, apply team thinking in a structured way to get a rapid and very deep understanding of the problem, and conducting a structured deselection of hypothetical causes to uncover the true root causes. What sets this approach apart from other is that it contains a guide of how to facilitate these steps. A case study was performed in a production department at Medicmeter to demonstrate its effectiveness and reproducibility. It resulted in a close to $60 \%$ reduction of the issue concerned.

Index Terms-Knowledge based systems, lean production, production engineering, production management, systematic problem solving, teamwork, quality management.

Managerial relevance - Production of new and advanced parts are often plagued by low yield which is accepted by management due to lack of experience with the parts in question. While yield increases as experience is gained it poses a significant risk as revenue is low or negative. Management should not accept low yield or wait for experience to form naturally. Low yield should be countered with an 
aggressive problem solving strategy. Problem solving is a special skill and not part of normal operations and it can be taught and managed. Management should perceive problem solvers as a specialist team that is assigned to problematic production area. Problem solvers are not technical experts. Problem solvers have tools and methods and are socially adept which allows them to work with the production team in question to solve problems. Systematic problem solving allows management to stabilize production and increase yield thereby creating economic feasible production. This paper presents one such approach to systematic problem solving.

\section{INTRODUCTION}

A Team of trouble-shooters have repeatedly managed to reduce error rates by $80 \%$ and increase productivity by $40 \%$ on average in production lines at Medicmeter. The team uses a systematic approach to problem solving with the following main components:

1. Extensive gathering of direct detailed process knowledge at the problem site

2. Applying team thinking in a structured way to get in-depth problem understanding

3. Conducting a structured deselection of hypothetical causes to uncover the true root causes

This approach has been used in mechanical production, assembly and chemical production, and appears to be general in nature. Needless to say, increasing quality has many benefits and may be a vehicle for reducing cost, increasing productivity and create further positive effects in the value chain. It has not been described before but is now named the NAX approach.

The importance of systematic problem solving in connection with production is well known. It is inherently difficult because it is a research activity where the relationship between cause and effect must be understood to improve the process. Employees close to the process hold the sufficient practical understanding of the production process to uncover this relationship but his group is not well versed in 
analysing the process nor has the time or influence at their discursion to embark on such a journey. This leaves many companies to accept low production yield because they lack a strategy to involve these employees and make use of their hands on knowledge.

There is no "gold standard" for solving problems systematically, but several methods are described in literature. The most common are the Eighth Disciplines of Problem Solving (8D), Define-MeasureAnalyse-Improve-Control (DMAIC) and the Shewhart-Deming Cycle Plan-Do-Check-Act (PDCA). These approaches provide instructive descriptions of the different steps of problem solving and on a general level, the methods contain the same elements, which are defining the problem, analysing the root cause and developing sustainable counter measures. Some methods suggest assembling a team for the problem solving process but for most parts it is considered a one-man's task. One thing these methods have in common is the absence of guidelines of how to apply them in practice and this gap in the literature was the incentive for writing this article.

The objective of this article is to present a case of systematic problem solving and based on this, formalize the NAX approach. The case was carried out in the sensor cassette department at Medicmeter where a quality issue was targeted. The same approach was applied for a different problem solving case at TransX , which is a US-based company that is also part of Medicmeters' parent Corporation. This case was omitted in this article for simplification but suggests that the method may be generally applicable - or at least across countries in the western culture. The case is outlined below followed by a thorough description the NAX problem-solving approach including a practical problem solving facilitation guide.

\section{LiteratURE REVIEW}

Most companies acknowledge the significance of problem solving and the subject has gained more attention the resent years. There is a substantial amount of literature on the subject, which further underlines the importance of it in relations to production. Searching for literature containing the words problem solving resulted in more than 330.000 articles, therefore the prefix systematic, logical, rational or 
practical was included in the search. Since the subject of this study is generated for the operational level of a company the word production was included in the search criteria.

The search resulted in following number of articles:

1. “Problem solving": 330.000 ;

2. "Systematic problem solving" AND production: 11;

3. "Logical problem solving" AND production: 2;

4. "Rational problem solving" AND production: 8;

5. “Goal directed problem solving” AND production: 3;

6. "Systematic problem solving" AND behaviour: 1;

7. "Problem solving and manufacturing": 4;

8. ”Problem solving and production”: 13;

The inclusion of the prefixes greatly decreased the literature available but was found justifiable since the main feature of the NAX approach presented in this article is the systematic way of solving problems. Based on this search 15 articles were selected for review and 23 were omitted since they did not contain any information on problem solving methods or behaviour. The pertinent articles where reviewed with the purpose of identifying whether there existed a general agreement about how to conduct a systematic problem solving process and if any of these articles contained a description of how to use the approaches described.

Since the book The Machine That Changed the World: The story of lean production came out in 1991, production companies worldwide have tried to imitate Toyota's manufacturing approach. Very few companies outside Toyota manage however to obtain the same extraordinary results as they do. Badurdeen and Gregory [9] claim that the reason for this ineffectiveness is that the companies are able to copy the tools and methods but not the mindset and culture of lean management. Marksberry, Bustle, and Clevinger [1] come to the same conclusion when they look into Toyota's 8-step problem-solving approach by 
investigating how managers are trained to support problem solving. They argue that other companies that have adopted the Toyota method use it as a template instead of a way of thinking which accounts for the low success rate outside Toyota. Linker and Meier [2] address this issue in their companion to the book The Toyota Way where the culture of the process is presented. There is a vast amount of literature on Toyota's 8-step problem solving process [3], [4], and [10]. They all describe the importance of defining the problem, conducting a root cause analysis and applying the PDCA cycle, but all lack guidance of how to use these methods. Shook [5] describes the A3 management process, which is core to the Toyota system. He outlines a mentoring process of a problem solving facilitator. This book describes how to apply the problem solving techniques but does not encompass any form of team assembling. Pokras [7] offers the only actual guide of how to apply the problem solving tools presented. It is a fairly detailed and applicable manual for the problem solving facilitator. Pokras recognises the strength of team thinking but pays no attention to the importance of spending time at the actual site of the problem and observing the process first hand. Ghosh [8] on the other hand emphasizes the importance of going to Gemba, which means place of action in Japanese, for deep problem and process knowledge in his case study of A3 problem solving implementation. The different problem solving tools are thoroughly described in a vast number of articles and books but the how-to element is missing. All in all there is a great gap in the literature when it comes to offering a practical and concrete problem solving facilitation guide. For this reason this article aims to provide such a guide.

\section{Sensor CASSETte CASE}

The NAX approach was applied in two separate problem-solving cases carried out in in Denmark and USA. In addition to these cases the author attended several problem-solving processes lead by others. For simplification the case carried out in Denmark will be presented in the article but a few examples from the USA case are introduced as well to illustrate key points. Same method and behaviour techniques were applied in both cases. These were selected because they had been applied by a team of problem solving 
experts, who on average managed to reduce error rates by more than $80 \%$ in relatively short time periods. This team had apparently found a way to systematically solve problems across a wide variety of production contexts - although the method was not formalized nor described.

The case presented in this article was carried out in the sensor cassette production department at Medicmeter in the capital region of Denmark. Medicmeter employs 2,500 people worldwide, has a turnover of approx. 600 million USD (2012) and it is a part of a US-based Corporation. Medicmeter is a leading provider of solutions for blood gas analysis, transcutaneous monitoring and immunoassay testing. There is a constant focus on quality in the company and the sensor cassette department is no exception. Here sensor cassettes (SC) used for blood gas analysis are produced and since the product is utilized in the industry it is very important that it is consistent and reliable.

There is a vast number of quality assuring steps in the production of the sensor cassettes. One of these is visual inspection with membranes embedded. Here about $12 \%$ of the intermediate product is discarded which results in a loss of 178.500 USD per year. In addition to the economic aspect this issue increases the pressure on the operators since they have to increase their workload to compensate for the discarded products.

The product stream was followed closely for approximately two weeks where each step in the SC production was thoroughly inspected for in-depth process understanding. The operators in the department were key actors in the observation period and offered great insight into both product and process. During the two weeks they were asked to photograph every discarded SC detected during the visual inspection. The visual display of the different defects ensured everyone had the same reference point and eased the classification of errors into five the five categories listed in Figure 1.

To evaluate the occurrence and thereby the relative importance of the error types, a form was designed in collaboration with the operators where they were to note every error found. Based on this data a pareto chart revealed that the particle/fibre category was by far the most critical one and accounted for $63 \%$ of the 
discarded SC and thus over 7\% of the total SC produced.

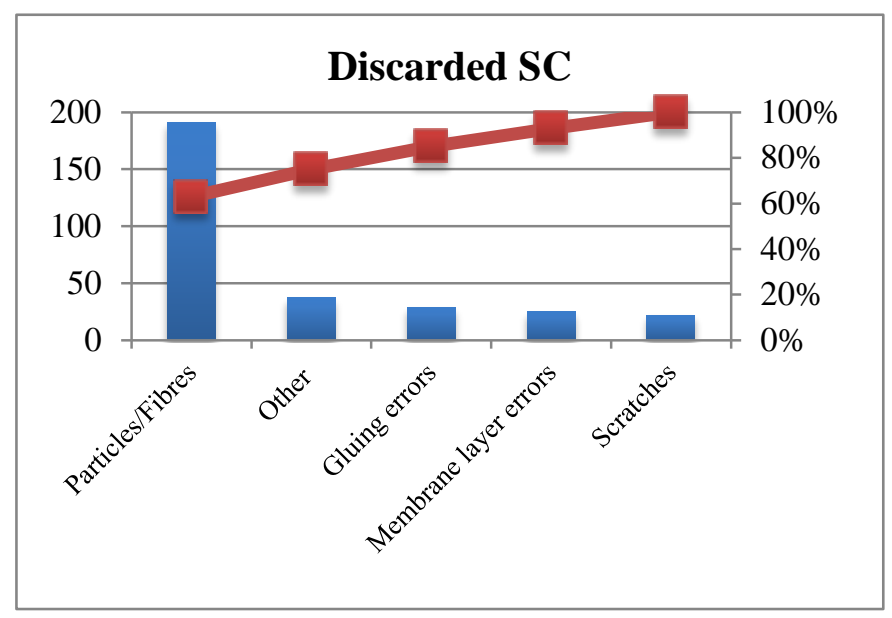

Figure 1: Pareto chart of the five error categories. The particle/fiber error accounts for $63 \%$ of the total discarded SC.

A problem solving team was assembled consisting of a discussion leader, who is the author of this article, two operators and the department coordinator. The operators most familiar with the production process were chosen for the task and the coordinator was included since he had extended knowledge of the process and was able to evaluate which countermeasures were possible to execute. This team held a root cause analysis (RCA) meeting, which took place on a whiteboard near the production area.

On the top of the board the main problem was written, which in this case was the large amount of discarded sensor cassettes with membranes. The five categories from the pareto chart were scrutinized one at a time and the course of the inquiring was led by the author of this article.

An example of the scrutinizing process is the analysis of the gluing error as shown below:

1.Discussion leader (DL): Why are so many SC with membranes discarded?

2. Team: Because the glue is not applied optimally

3.DL: Why is the glue not applied optimally?

4. Team: Because the glue is spurted out of the gluing gun 
5.DL: Why is the glue spurted out of the gluing gun?

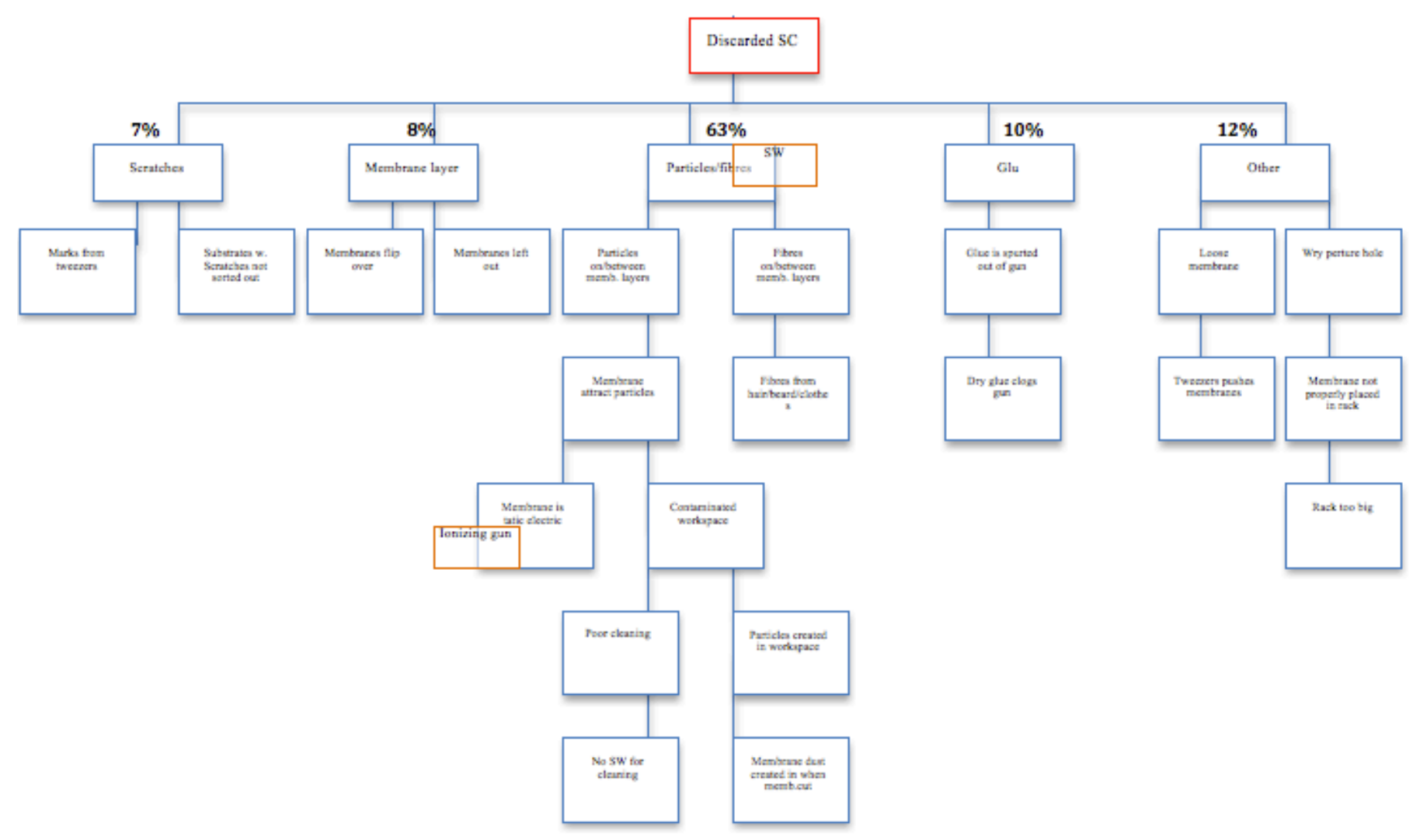

Figure 2: The RCA tree from the SC case. The red box contains the problem, the blue boxes the potential causes and the orange the actions

Figure 2 shows the RCA tree where the input from the team is outlined on the Caused by cards. Since the particle/fibre category accounted for $63 \%$ of all errors, this was chosen as the place to intervene. The following causes were chosen for further investigation: contaminated workspace and the static electricity of the membrane material. The membranes were handled in an open workspace which the team thought increased the risk of particles/fibres ending up on or between the membranes. The static electricity of the membrane material was believed to attract the 
particles/membranes. These causes were chosen since their influence was easy to test and would likely have a big impact on the yield.

To test these hypothetical causes the team chose a few actions to carry out. The membrane process was to be moved into a controlled workspace with air exhaustion and a standard work (SW) procedure for preparation and cleaning of this space was to be introduced, these are shown in orange in Figure 2.

This should ensure consistent working methods when handling the membranes and minimize the particles/ fibres near the process. The procedure included cleaning the area with a deionization gun and alcohol wipes. The de-ionization blew the particles into the air where the air ventilation system was supposed to remove them. The final action was to blow ionized air onto the membranes to counteract their static electricity. All actions were planned in cooperation with the operators to ensure the steps were manageable.

The operators were asked to keep documenting the errors found during visual inspection to survey the impacts of the actions introduced. Surprisingly the changes increased the occurrence of particle/fibre errors. The team went back to the process and found that the operators did not turn on the air ventilation when handling the membranes or cleaning the workspace. This explained the increased number of discarded SC because the de-ionization gun whirled the particles/fibres into the air and since the air ventilation system wasn't on they weren't removed and eventually ended up in/on the membranes. To assure this would not repeat itself a requirement for turning on the air system was added to the SW procedure, which resulted in a reduction of particle/fibre errors. The overall outcome of this systematic problem solving process was a $7 \%$ to $3 \%$ reduction of errors resulting in a 59.500 USD saving pr year. 


\section{Development of the Nax Problem Solving Approach}

The approach for solving the problem in the abovementioned case will be thoroughly analysed below including facilitation guidelines. The objective of the section is to serve as a practical and concrete guide for solving problems systematically. The main steps of the process are to gather direct detailed process knowledge at the problem site, breaking down the problem via visual management and data analysis, assembling the right team, generating team thinking and conducting a root cause analysis with rapid repetitive cycles of deselection of hypothetical causes with the aim of selecting the best possible countermeasure.

\section{1)Gather Direct Detailed Process Knowledge}

\section{A. Spend time at the process}

The importance of spending time near the process cannot be stressed enough. It is emphasised in almost all problem solving literature, however very few do it in practice, at least to the necessary extend. Liker [22] describes "standing in the chalk circle” which is a reference to Taiichi Ohno's exercise where a manager is told to stay in a chalk circle drawn on the production floor to understand and observe at the actual place. Going to Gemba is a widely used expression but the meaning varies. In this article it signifies spending time directly at the process, which is essential for gathering information from where the problem originates and getting detailed process knowledge. A rule of thumb of how much time should be spent at Gemba is to stay within an arm's length from the process until you are bored - and then double up the time. The ability to intercept information greatly increases when longer periods are spent observing. It is like when entering a dark room you cannot see but if you stay there for an extended time your eyes gradually adapt to the environment and you gain the ability to notice more and more details. The problem solving team should go to Gemba to reach the same level of process insight and a joint 
reference point. This greatly increases the quality of the RCA meeting because many potential causes can be eliminated beforehand. A good example of this occurred in the case where one team member thought the fibres found between the membranes originated from the operators' hair, but when the team went to Gemba they learned that the operators wore hairnets in the production area. The cause wasn’t brought up at the RCA meeting since it wasn't relevant.

\section{B. Facilitation}

People facilitating problem solving often have a questioning mind by nature. However this is not the case for everyone and the facilitators' main objective is to get the team on board in generating in-depth process understanding collectively. It is vital to be physically and mentally present at Gemba. This signals engagement and interest in the project and the operators work in general. Including the operators working in the problem area is essential because of their intricate knowledge and their input should be acknowledged since they know the product and process inside out.

The following points are very useful when going to Gemba [7].

1. Announce agenda and intention of the stay in the production area;

2. Ask questions to get the group thinking, repeat and rephrase when necessary;

3. Make pauses when asking to let the questions sink in;

4. Introduce new perspectives into ongoing discussion;

5. Praise every input regardless of its quality since it will encourage the operators to come forward with their thoughts and hypotheses;

6. Ask for help, set the standard and thereby make the operators feel appreciated;

\section{Visualise the Problem}

Visualisation is a powerful tool for gathering detailed process knowledge from Gemba. Photographs allow people to discuss what they see and not what they think they see and create a 
common perception of the situation. In some situations the extent of a problem can be hard to evaluate. In these situations multiple photographs of the faulty subject(s) can bring new understanding of the issue. Arranging the errors into categories only based on visual features aids the team in assessing the magnitude of the problem and clarifies where to centre the attention at the RCA meeting. Sometimes it is necessary to utilize ancillary equipment for visualising items that cannot be seen with the bare eyes. In the SC case the operators used a microscope camera for showing the particles. The photographs revealed that they were white and had the same structure and therefore it was fair to assume they originated from the cut-off membrane material. Linker and Meier [14] agree on the importance of visually depicting the problem, but focus on graphical presentations in the form of charts, not actual photographs. Another approach is to gather video material to visualise the process in movement.

\section{Gain Deep Problem Knowledge}

Creating an overview of the extent of a problem by means of data is essential preparation for the RCA meeting. Pareto charts are often the weapons of choice for this purpose since they are easy to make and highlight the most important types of errors and ensure that the most critical issues are brought into focus. This can e.g. be the most occurring type of errors or the most common source of defects. This overview oftentimes prevents time consuming debates about where to intervene since consensus is often created within the team. In the SC case it was apparent from the pareto chart that the particle/fibre issue was the most critical one and since it accounted for $63 \%$ of the discarded products it was chosen as the starting point.

\section{E. Incentive}

When presenting the data at the RCA meeting it is often helpful to translate the data into something of value for the different team members. Instead of explaining to the operators that the 
reduction of particles would increase the yield X \% they might be more encouraged to take part if they knew that this same reduction would result in a $\mathrm{X} \$$ saving. It is often easier to comprehend data presented in man-hours or money.

\section{2)Team Thinking - How to Make a Group of People Think Together Systematically}

Problem solving is not a one-man show - it is a team sport. Gathering the right people and making full use of their competences is vital for the successful problem solving.

\section{A. Team Composition}

The composition of the team is alpha and omega. Gathering a number of bright people in a room and writing Kaizen on the door is not enough, there is more to it than that.

In every team there should be a problem facilitator. This person is responsible for assembling the team and is the one to lead the discussion at the RCA meetings. In most cases it is advantageous to include the operators working in the production department. They know the process in detail and though they might not have the academic background for a deep understanding of the underlying causes their hands on knowledge is invaluable. For particularly complex technical problems input from specialists can be necessary for in-depth understanding of the problem. These specialists are typically R\&D engineers or other with extended process, design or product knowledge.

Two common pitfalls of team assembling are wrong proportion of operators to specialists in the team and the exclusion of operators.

When the proportion of operators to specialists is off, the teams' potential is not utilized optimally. One such example took place at a RCA meeting in the TransX case previously mentioned. In the beginning of the meeting three operators and two engineers were present and 
everyone contributing to the analysis. As the meeting preceded four more engineers/managers joined. Being in minority obviously made the operators uncomfortable and they stopped offering their input to the discussion of the problem. It seemed like they were intimidated by the presence of their superiors and lacked the confident to come forward with their knowledge and ideas. Teams lacking members with hands on knowledge miss out on valuable process literacy. Managers rarely spend time at Gemba and do therefore not have extensive knowledge about the processes. When they perform root cause analysis they often see the symptoms of the problem instead of the actual problem. Operators on the other hand often see the causes since they are in close contact with the product and process

3)Structured Deselection of Hypothetical Causes

Figure 3. Centre team's focus on one subject at a time

This section outlines how to make a group of people systematically think through a complex problem, systematically evaluate potential cause(s) and systematically select appropriate counter measures by means of rapid PDCA cycles.

\section{A. Root Cause analysis meeting}

The overall objective of the RCA meeting is to get a deep understanding of the problem, elaborate on possible causes and finding an optimal counter measure with information gathered at Gemba This is done in a systematic and visual manner where the team members' attention is centred on one subject at a time as demonstrated in Figure 3. This is pivotal for full employment of their competences since the team members can build on each other’s ideas and thoughts. 


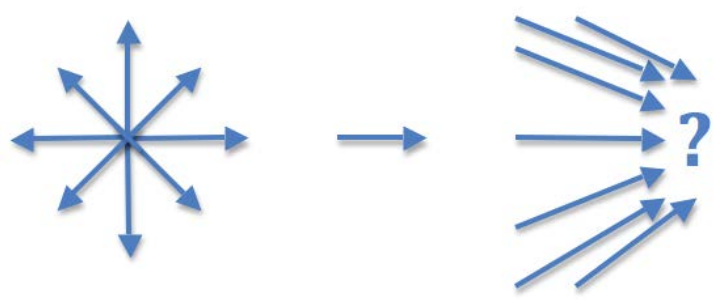

Figur 3: The team focuses on one subject at a time.
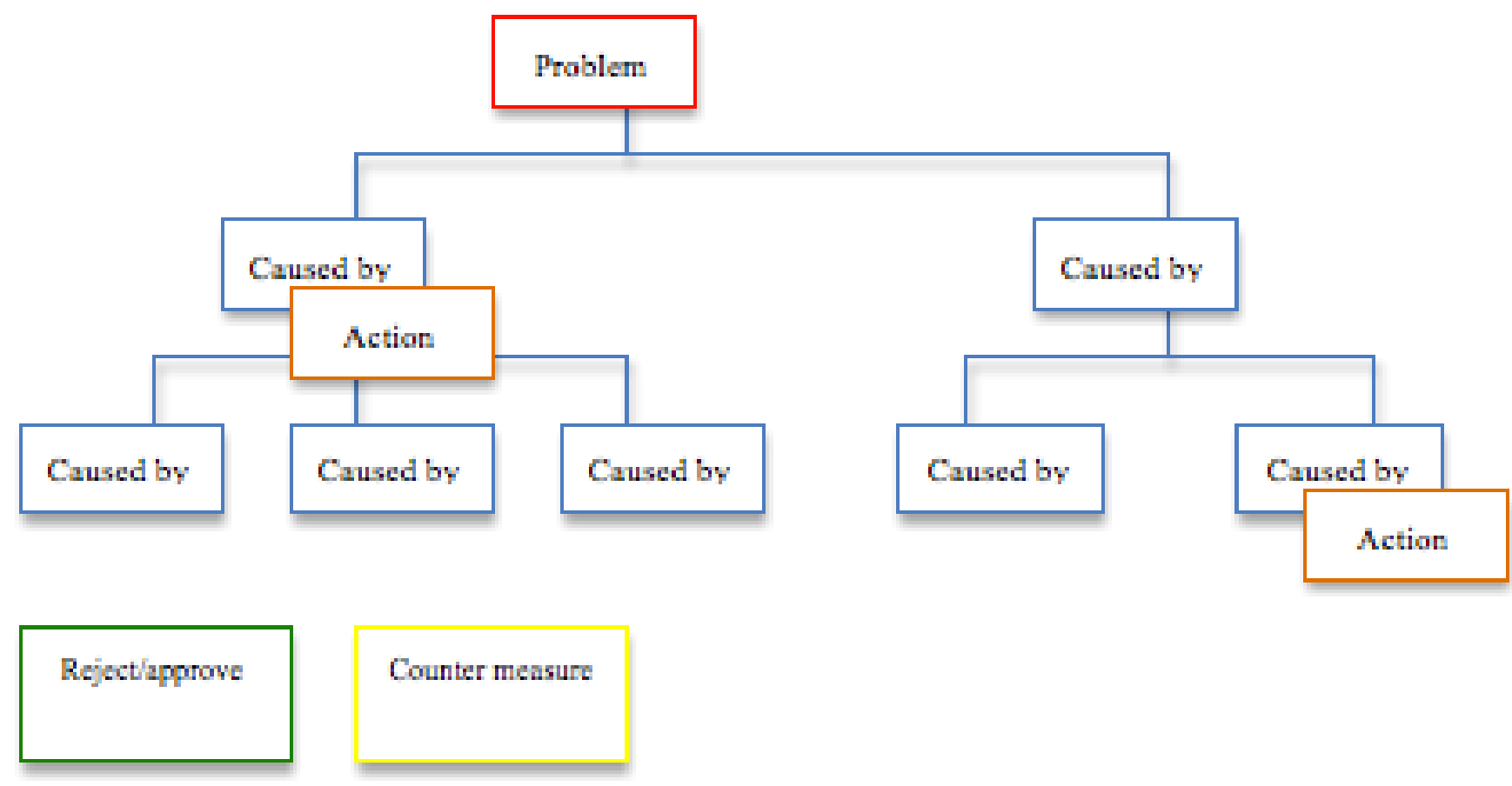

Figur 4: A schematic presentation of a RCA tree.

When starting the meeting the problem statement is written on the Problem card after which the facilitator writes the suggested possible counter measure on the Caused by cards. A version of $5 \mathrm{x}$ why [13] is applied for every possible cause and the facilitator challenges the team 
members by asking why until they feel further elaboration is not needed or until more information is required about the subject from Gemba. The Caused by cards are the only type of cards used in the beginning to assure the causes are fully understood before counter measures are chosen. This furthermore ensures that the RCA meeting is actually an analysis meeting and not a meeting where team members debate on which counter measures to try out.

A schematic presentation of a RCA tree is found in Figure 4. The meeting should not exceed a time cap of approximately one hour since it is a mentally exhausting session and people are more likely to jump to conclusions and making irrational decision when tired. Before ending the meeting the team should make an action plan of the next steps of the problem solving process.

\section{B. RCA Toolbox}

The RCA tree used for organizing the causes in causal chain (explained below) and for this purpose five different cards are prepared for the RCA meeting. A vast number of Caused by cards should be made, some Approved/Rejected cards as well as Action cards, few Countermeasure cards and one Problem card. The reason for this distribution is that when searching for the appropriate root cause many potential causes have to be investigated. A few of these are chosen for further investigation thereby requiring an Action card. The conclusion of the investigation is written on the Approved/Rejected card depending on the output. When the root causes have been identified they will be given a suitable counter measure. The cards are colour coded for visual aid and they should be movable since it is often necessary to rearrange them as the analysis proceeds.

Only tangible statements can be written on the "Caused by" cards. When a problem occurs, something specific has happened and for finding an optimal countermeasure the cause has to be clearly identified. It is hard to find a suitable counter measure for an unspecific cause. An 
unspecific statement from the SC case could e.g. be "varying amount of glue is dispensed” but a concrete one would be "too much glue is dispensed" or "too little glue is dispensed".

\section{Causal Chain}

The root cause analysis tree is the primary constituent of the RCA meeting where all thoughts and hypotheses regarding the problem are gathered systematically. The causes are organized in causal chain where one cause in the chain causes the next [13]. The main benefit of this arrangement is that it allows for exhaustive analysis where the goal is to find the primary root cause(s). It also ensures that all causes are directly linked to the problem thereby eliminating the possibility of sidetracking. This procedure greatly helps in finding the actual cause.

\section{Facilitation}

The facilitators' most pivotal task during the RCA meeting is to steer the discussion towards one subject at a time and avoid side tracking. In other words the facilitator is to eliminate the storm in the brainstorming concept. The facilitator should keep his ideas to himself, stay objective and resists the urge to offer an opinion since his role is to direct the participation and not be the main provider of input. Operators tend to believe the facilitator has more insight into the problem and are likely to agree on his contribution instead of actually making up their own mind on the subject.

One of the main pitfalls of the RCA meeting is sidetracking. It is reasonable to assume the team members have a favourite cause and maybe even counter measure prepared prior to the meeting. In these cases they are likely to focus more on marketing or defending their favourite cause than looking into other options. Gadd [6] calls this "my ugly baby" solution since people tend to like their own creations and oversee their shortcomings while they might be obvious to others. This issue has to be addressed right away and a way of doing so is to acknowledge their proposals 
when starting the meeting, writing them all on a Caused by card and promise to look into them during the meeting. The same tactic goes for when members suggest new topics during an ongoing discussion of another cause.

The following points shall be kept in mind when leading the discussion at the RCA meeting.

1. Introduce the objective of the meeting;

2. Establish a realistic time frame, stick to the agenda and keep discussion on track;

3. Focus on processes and not people when proposing potential causes;

4. Restate topic to focus the group on one subject at a time and repeat the whole chain of causes to keep relation to the problem;

5. Steer discussion with Caused by cards and avoid debating counter measures too soon;

6. Reword all suggestions to concrete statements before writing them on cards;

7. Clarify meanings, rephrase questions when needed and restate important information;

8. Praise all ideas being brought up, even the bad ones;

9. Use silence effectively and wait out pauses to give the team time to think;

10. Encourage everyone to participate, draw out quiet members and call out those with something to say;

11. Avoid interfering with interactions but cut in if the tone gets harsh;

12. At the end of the meeting, summarize what has been accomplished and obtain consensus about the next step;

\section{E. Rapid-PDCA Cycles}

The cycle of planning, doing, checking and acting (PDCA) is an iterative process consisting of a cascade of short meetings with information seeking at Gemba in-between [15] and [16]. The prefix rapid is added to the PDCA cycle since each step should be quick and relatively easy to 
conduct. The aim of the cycle is to collect information about the hypothetical causes to either approve or reject them.

Følledal, Walløe, and Elster [11] describe this process of testing hypotheses via their empirical consequences, whose effect can be determined via direct observation. When consensus has been made at the RCA meeting about which causes to focus on the team decides on a few actions to perform at Gemba. The actions can either be in the form of small tests where solutions are tested in controlled environment to determine if a variable, influences the process positively, or merely observing the process again.

When the evidence has been collected the team returns to the RCA tree for further analysis and based on the newly gathered information they are able to rule out or highlight some causes. The facts and evidence obtained can park bad solutions and averts discussions about favourite causes. The PDCA cycle is repeated until enough evidence has been obtained for an informative identification of the root causes and suitable counter measures have been identified. In the SC case the PDCA steps were conducted as well as an extra cycle of DC. In some cases many rounds of the cycle is needed for finding the optimal counter measure while some only require one.

\section{DISCUSSION}

This study adds to a growing literature stream that lauds the importance of problem solving. The aim of this paper is to present a case of systematic problem solving and outline the NAX approach based on this. The NAX approach is an extraordinarily effective systematic problems solving framework and facilitation guide developed by a group of problem solving experts at Medicmeter . 
The application of the framework was empirically studied in the case to demonstrate its effectiveness and reproducibility in production context. This process resulted in a reduction of the defects concerned from $7 \%$ to $3 \%$ which yields a 59.500 USD saving pr year. This result was not surprising since the approach has shown to create exceptionally results in several different departments at Medicmeter . One thing that is important to mention is that the Danaher companies setting the stage for the problem solving cases both have a high level lean culture which may have eased the conducting of the problem solving processes. The operators working in the production departments are used to bringing forward their input and accustomed to a constantly changing environment caused by the frequent problem solving processes conducted.

It is not possible to generalise about the cost of introducing the NAX approach. The method is resource demanding as it requires both time and manpower. The time needed for solving a problem varies from case to case and it cannot be estimated beforehand. This is the main reason for the difficulty of evaluating the cost of the process since a long course requires considerably more resources than a short. Not all companies have the capability of having employees dedicating their time to problem solving on a daily basis. In the production department at Medicmeter there are two problem solving facilitators, who account for $17 \%$ of the departments' workforce, that spend approximately half their time dealing with problems. Needless to say that is not inexpensive. Removing a number of operators from the standard production is also costly. On the other hand, unsolved problems and quality issues are very costly although many companies have unsolved problems for years and merely accept the low production yield. Unsolved problems do not stop being an expense until they are solved and therefore efforts for solving them are almost always advantageous. 
The NAX problems solving approach is considered feasible sine it has an impressive track record of reducing error rates by more than $80 \%$ and raise the productivity by $40 \%$ on average. The result of the SC case supports this tendency though the reduction is not as extensive. There are still ways to improve the production process, thereby further reducing the error rates, and many of the potential causes are yet to be investigated. A great side benefit of the problem solving effort is that more and more knowledge is gathered concerning the product and process. Each session establishes new literacy, which can turn out to be very valuable for e.g. future product or process development.

\section{REFERENCES}

[1] P. Marksberry, J. Bustle, and J. Clevinger, (2011) Problem solving for managers: $a$ mathematical investigation of Toyota's 8-step process. Journal of Manufacturing Technology Management,Vol. 22 Iss: 7, pp.837 - 852

[2] J. Liker, and D. Meier, The Toyota Way fieldbook. New York, NY: McGraw-Hill, 2005.

[3] M. Rother, Toyota Kata: Managing people for improvement, adaptiveness and superior results. New York, NY: McGraw-Hill, 2010

[4] J. Liker, and M. Hoseus, Toyota Culture: The heart and soul of the Toyota way. New York, NY: McGraw-Hill, 2008.

[5] J. Shook, Managing to learn: using the A3 management process to solve problems, gain agreement, mentor and lead. Cambridge, Ma: Lean Enterprise Institute, 2010

[6] K. Gadd, TRIZ for Engineers: Enabling Inventive Problem Solving. West Sussex, UK: John Wiley \& Sons. pp. 52, 2011 
[7] S. Pokras, Systematic problem-solving and decision-making. Los Altos, CA: Crisp publications INC, pp. 20-21, 1989

[8] M. Ghosh (2012) A3 process: A pragmatic problem-solving technique for process improvement in health care. Journal of health management. 14 (1), pp 1-11.

[9] F. Badurdeen and B. Gregory, The soft side of lean: Analyzing corporate culture can point the way to necessary changes. Industrial Engineer: IE, 2012. 44 (2) pp 49

[10]C. Soltero, Creating an adaptable workforce: Lean training and coaching for improved environmental performance. Environmental Quality Management, 2011. 21 (1) pp 9

[11]D. Følledal, L. Walløe, and J. Elster, Begrundelse og metode i naturvidenskab in Politikens bog om moderne videnskabsteor, Copenhagen, DK: Politikens Forlag A/S, 1992

[12]J. Liker, and D. Meier, The Toyota Way fieldbook. New York, NY: McGraw-Hill, pp. 61, 2005

[13]J. Liker, and D. Meier, The Toyota Way fieldbook. New York, NY: McGraw-Hill, pp. 342346, 2005

[14]J. Liker, and D. Meier, The Toyota Way fieldbook. New York, NY: McGraw-Hill, pp. 349351, 2005

[15]M. Rother, Toyota Kata: Managing people for improvement, adaptiveness and superior results. New York, NY: McGraw-Hill, pp.142-155, 2010.

[16]J. Liker, and D. Meier, The Toyota Way fieldbook. New York, NY: McGraw-Hill, pp. 364375, 2005 